Supporting Information for manuscript

\title{
Competitive coadsorption dynamics of viruses and dissolved organic matter to positively charged sorbent surfaces
}

\author{
Antonius Armanious ${ }^{1,2}$, Melanie MünCh ${ }^{1}$, Tamar KOHN $^{2}$, Michael Sander ${ }^{1, *}$ \\ 1: Institute of Biogeochemistry and Pollutant Dynamics (IBP) \\ Department of Environmental Systems Science, ETH Zurich, Switzerland \\ 2: Laboratory of Environmental Chemistry, School of Architecture, Civil and \\ Environmental Engineering (ENAC), École Polytechnique Fédérale de Lausanne \\ (EPFL), Lausanne, Switzerland
}

Submitted as manuscript to Environmental Science \& Technology

* Corresponding author: Michael Sander

email: michael.sander@env.ethz.ch

Phone: $+41(0) 44632-8314$

Fax: $+41(0) 44633-1122$

Number of Figures: $\quad 11$

Number of Tables: $\quad 03$

Number of Pages: $\quad 33$ 


\section{S1. Materials and Methods}

\section{S1.1 Chemicals}

Cysteamine, Bis(2-hydroxyethyl)amino-tris(hydroxymethyl)methane (BISTRIS) were obtained from Sigma, tris(hydroxymethyl)aminomethane (TRIS) from Aldrich. Anhydrous ethanol, ethanol, acetic acid, and sodium hydroxide were from Fluka, and sodium chloride $(\mathrm{NaCl})$ and hydrochloric acid $(\mathrm{HCl})$ were from Merck. All chemicals were of analytical grade and used as received.

\section{S1.2 Virus propagation and purification}

Bacteriophages MS2 (DSMZ 13767), Q $\beta$ (DSMZ 13768) and the virus host Escherichia coli (E. coli) (DSMZ 5695) were purchased from the German Collection of Microorganisms and Cell Cultures (Braunschweig, Germany). Bacteriophage fr (ATCC-15767-B1) was obtained from LGC Standards (Molsheim, France), and bacteriophage GA was kindly provided by Dr. Joan Jofre (University of Barcelona). The viruses were propagated and purified as detailed in Armanious et al. (2016). ${ }^{1}$ In short, the viruses were separately propagated in the above E. coli strain during incubation in LB media for 5 hours at $37{ }^{\circ} \mathrm{C}$. The bacterial cells were subsequently lysed with chloroform and centrifuged at $4000 \mathrm{~g}$ for $15 \mathrm{~min}$ to remove bacterial debris. The supernatant was collected and purified by repeated washing through 100 kDa Amicon Ultra-15 centrifugal filters (Millipore, Billerica, MA, USA) using virus dilution buffer ( $5 \mathrm{mM} \mathrm{HPO}_{4}{ }^{2-}, 10 \mathrm{mM} \mathrm{NaCl}, \mathrm{pH}$ 7.4). To this end, approximately 10 $15 \mathrm{~mL}$ of dilution buffer were re-added to virus-containing retentate left in the filter after each centrifugation step, followed by re-centrifugation. The final retentate in the 
filter was sterile filtered through $0.1 \mu \mathrm{m}$ syringe filters to yield the virus stock solutions. The stock solutions were stored in the dark at $4^{\circ} \mathrm{C}$ until use.

Directly before adsorption experiments, an aliquot of the respective virus stock solution was again repeatedly washed as described above using $100 \mathrm{kDa}$ Amicon Ultra-15 centrifugal filters for a total of at least ten cycles. During this purification step, a slightly different washing solution ( $3 \mathrm{mM}$ Tris, $10 \mathrm{mM} \mathrm{NaCl}, \mathrm{pH} 8.0$ ) was used. The final volumetric washing ratios of this second step ranged from 500 to 2000. Finally, the filter retentate was diluted in $\mathrm{pH} 6$ buffer solutions to final virus concentrations of $\approx 5 \cdot 10^{11}$ virion $\cdot \mathrm{mL}^{-1}$ for MS2, fr, GA, and Q $\beta$.

This virus purification protocol resulted in highly reproducible adsorption data. We previously showed that shortened purification protocols or the use of polyethylene glycol in the purification process resulted in irreproducibility of adsorption in singleadsorbate systems. ${ }^{1}$

\section{S1.3 DOM solutions}

Stock solutions of SRHA and SRNOM were prepared by dissolving the respective DOM in MilliQ water to final concentrations of $500 \mu \mathrm{g}_{\mathrm{DOM}} \cdot \mathrm{mL}^{-1}$. Following dissolution, the stock solutions were adjusted to $\mathrm{pH} 7$ using $1 \mathrm{M}$ $\mathrm{HCl} / \mathrm{NaOH}$. The solutions were then left to equilibrate overnight at $4^{\circ} \mathrm{C}$. The solution $\mathrm{pH}$ values were subsequently re-measured and, if values had drifted, adjusted a second time to $\mathrm{pH}$ 7. Finally, the solutions were sterile filtered $(0.2 \mu \mathrm{m})$, divided into smaller aliquots, and stored at $4^{\circ} \mathrm{C}$ in the dark. 


\section{S1.4. Formation of amine-terminated self-assembled monolayers}

A detailed protocol for forming the aminoalkylthiol self assembled monolayers (i.e., SAM-NH${ }_{3}^{+}$) on gold-coated QCM-D sensors (QSX 301, QSense) is provided in Armanious et al. (2014). ${ }^{2}$ In short, the sensors were first thoroughly cleaned in three consecutive steps: (i) sonication for $15 \mathrm{~min}$ in a solution of sodium dodecyl sulfate ( $2 \%$ by weight), followed by rinsing with MilliQ water and drying in an air stream; (ii) treatment of the dried sensors in a UV/ozone chamber (Bioforce Nanoscience) for 30-40 min; and (iii) applying a few droplets of Piranha solution to the sensor surface (3:1, concentrated $\mathrm{H}_{2} \mathrm{SO}_{4}: \mathrm{H}_{2} \mathrm{O}_{2} 30 \%$; Note: Piranha is a very aggressive oxidant and should be handled with uttermost care) for two minutes. The sensors were subsequently washed in MilliQ water and ethanol and dried with $\mathrm{N}_{2}$ flow. The cleaned sensors were then reacted with cysteamine in anhydrous ethanolic solutions (0.5-2.0 $\mathrm{mM}$ cysteamine) for at least eight hours. This reaction was conducted in an $\mathrm{O}_{2}$-free glove box (pure $\mathrm{N}_{2}$ atmosphere) to rule out oxidation of the aminoalkylthiols. The SAM- $\mathrm{NH}_{3}{ }^{+}$surface-modified sensors were subsequently rinsed and sonicated in ethanol to remove unbound alkylthiols. The sensors were used in adsorption experiments on the same day of preparation. The successful formation of $\mathrm{SAM}^{-\mathrm{NH}_{3}}{ }^{+}$ surfaces was previously verified by ellipsometry and contact angle measurements. ${ }^{2}$

\section{S1.5. Types of adsorption experiments}

Table S1 shows the sequence by which solutions were delivered to the QCMD flow cells in the three types of adsorption experiment conducted in this study. Type

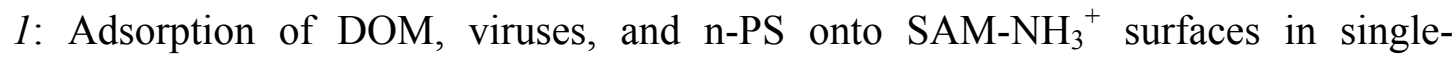


adsorbate systems. These experiments consisted of three successive steps: (i) equilibrating the $\mathrm{SAM}-\mathrm{NH}_{3}{ }^{+}$surfaces by running adsorbate-free solutions over the sensors to obtain stable $\Delta f_{\mathrm{n}} / \mathrm{n}$ and $\Delta D_{\mathrm{n}}$ readings; (ii) delivering solutions containing either DOM, virus, or n-PS over the sensors until adsorption plateaued or, for systems with slow and continuous adsorption, until the entire prepared solution was delivered; (iii) rinsing with adsorbate-free solutions to assess adsorption reversibility. Type 2: Adsorption of viruses and n-PS onto DOM adlayer surfaces in single-adsorbate systems. These experiments consisted of five successive steps; steps (i) to (iii) were analogous to the above-described steps except that step (ii) was conducted with DOM only, resulting in the formation of DOM adlayers on the $\mathrm{SAM}_{\mathrm{NH}}{ }^{+}$surfaces. $^{2}$ The two additional steps were: (iv) delivering solutions containing either one of the viruses or n-PS over the DOM-adlayers until adsorption plateaued or the entire prepared solution was delivered; (v) rinsing with adsorbate-free solutions. Type 3: Adsorption of viruses and n-PS onto $\mathrm{SAM}-\mathrm{NH}_{3}{ }^{+}$surfaces in DOM coadsorbate systems. These experiments involved the same three steps (i) to (iii) described above only that the solution used in step (ii) contained both of the respective coadsorbates.

Table S1. Sequences by which solutions were delivered to the QCM-D flow cells in the three types of adsorption experiments. For each experiments, all solutions had the same $\mathrm{pH}$ and ionic strength.

\begin{tabular}{|c|c|c|c|c|c|c|}
\hline & System & $1^{\text {st }}$ solution & $2^{\text {nd }}$ solution & $3^{\text {rd }}$ solution & $4^{\text {th }}$ solution & $5^{\text {th }}$ solution \\
\hline Type 1 & single-adsorbate & $\begin{array}{l}\text { adsorbate-free } \\
\text { solution }\end{array}$ & $\begin{array}{l}\text { solution with } \\
\text { one virus or n- } \\
\text { PS or DOM }\end{array}$ & $\begin{array}{l}\text { adsorbate-free } \\
\text { solution }\end{array}$ & - & - \\
\hline Type 2 & single-adsorbate & $\begin{array}{l}\text { adsorbate-free } \\
\text { solution }\end{array}$ & $\begin{array}{l}\text { solution with } \\
\text { DOM }\end{array}$ & $\begin{array}{l}\text { adsorbate-free } \\
\text { solution }\end{array}$ & $\begin{array}{l}\text { solution with } \\
\text { one virus or n- } \\
\text { PS }\end{array}$ & $\begin{array}{l}\text { adsorbate-free } \\
\text { solution }\end{array}$ \\
\hline Type 3 & coadsorbate & $\begin{array}{l}\text { adsorbate-free } \\
\text { solution }\end{array}$ & $\begin{array}{l}\text { solution with } \\
\text { one virus and } \\
\text { DOM or n-PS } \\
\text { and DOM }\end{array}$ & $\begin{array}{l}\text { adsorbate-free } \\
\text { solution }\end{array}$ & - & - \\
\hline
\end{tabular}




\section{S2. Results and Discussion}

S2.1 Sizes and charge of the polystyrene nanospheres.

The hydrodynamic diameters and the surface charges of the polystyrene nanospheres (n-PS) were determined based on dynamic light scattering (DLS) and pH-dependent electrophoretic mobility measurements, respectively, using a Zetasizer Nano ZS instrument (Malvern Instruments, Malvern, UK). The $\mathrm{pH}$ dependency of electrophoretic mobility was determined by varying the $\mathrm{pH}$ from 10 to 2 with $\Delta \mathrm{pH}=1$ using an autotitrator (model MPT-2; Malvern, UK) that delivered $0.1 \mathrm{M} \mathrm{HCl}$ and 0.1 $\mathrm{M} \mathrm{NaOH}$ titration solutions. All solutions used were filtered through $0.1 \mu \mathrm{m}$ syringe filters prior to use. Zeta potentials were calculated using the Zetasizer software (version 7.03; Malvern, UK).

Figure S1a shows the number distribution of n-PS at pH 6 and $I=10 \mathrm{mM}$. The n-PS had a very narrow size distribution with a mean size of $30.6 \mathrm{~nm}$. Figure S1b shows that n-PS had negative surface charges over the whole tested $\mathrm{pH}$ range and that the charges increased with increasing $\mathrm{pH}$. The extrapolated isoelectric point was approximately IEP $\approx 2$, in good agreement with previously reported IEP values of $\approx$ $2-3 .^{3}$ 
a. size distribution of $n-P S$

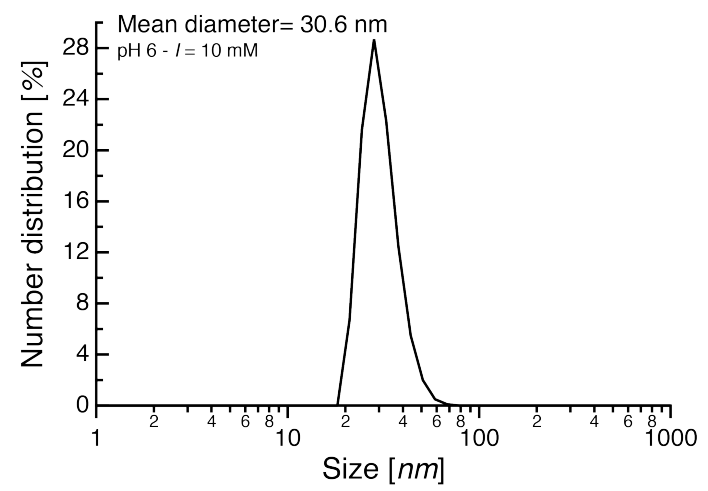

b. zeta potentials of the n-PS

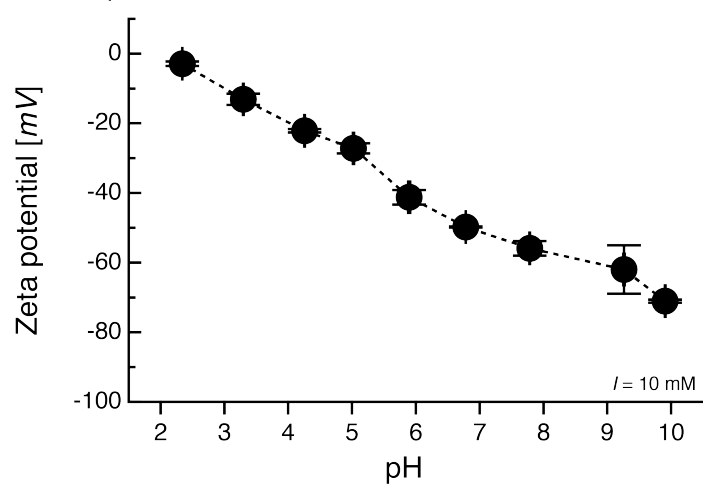

Figure S1. Size distribution and surface charges of the polystyrene nanospheres (n-PS). (a) Number size distribution of n-PS as determined by dynamic light scattering at $\mathrm{pH} 6$ and $I=10 \mathrm{mM}$, adjusted using $\mathrm{NaCl}$. (b) Zeta potentials of $\mathrm{n}$-PS as a function of solution $\mathrm{pH}$, determined by electrophoretic mobility measurements at $I=10 \mathrm{mM}$, adjusted using $\mathrm{NaCl}$.

S2.2 Viscoelastic properties of adlayers formed in virus single-adsorbate and virusSRHA coadsorbate systems

Figure $\mathbf{S 2}$ shows the adsorption profiles of MS2 (panel a), fr (panel b), GA (panel c), and $\mathrm{Q} \beta$ (panel d) to the $\mathrm{SAM}-\mathrm{NH}_{3}{ }^{+}$surfaces determined in the absence of SRHA (i.e., virus single-adsorbate systems; $0.00 \mu \mathrm{g}_{\mathrm{SRHA}} \cdot \mathrm{mL}^{-1}$ ) and in the presence of varying concentrations of SRHA as a coadsorbate. The data is re-plotted from Figures 2a-d in the manuscript but in the form of changes in resonance frequencies of the ninth overtones (i.e., $\Delta f_{9} / 9$ ) versus changes in the respective dissipation values (i.e., $\left.\Delta D_{9}\right)$.

In the initial stages of adsorption, all adsorption profiles collected in the single- and the coadsorbate systems overlapped. During these stages, large fractions of the $\mathrm{SAM}-\mathrm{NH}_{3}{ }^{+}$surfaces were unoccupied by adsorbates and hence available for adsorption of viruses and/or SRHA that approached the surface. The changes in the frequency and dissipation values were dominated by adsorbed viruses due to the much 
higher initial adsorption rates of the viruses (Figures $\mathbf{1 b , c}$ in the manuscript) and hence larger contributions to the total adsorbed mass, as compared to SRHA. In later stages of the experiments, the adsorption of MS2 and fr ended when the $\mathrm{SAM}^{-\mathrm{NH}_{3}}{ }^{+}$ surfaces became fully covered by the viruses (i.e., in the single-adsorbate system) or by both the viruses and SRHA (i.e., in the coadsorbate systems). The adsorption profiles collected in the MS2 and fr coadsorbate systems did not largely deviate from the adsorption profile determined in the MS2 and fr single-adsorbate systems. The overlap in the adsorption profiles suggest that the viscoelastic properties of the MS2 and fr adlayers formed in the presence of SRHA were mainly dominated by the dissipation of energy at the contact points between the viruses and the $\mathrm{SAM}^{-\mathrm{NH}_{3}}{ }^{+}$ surfaces. $^{4}$

Conversely, at later stages of adsorption, the adsorption profiles of GA and $\mathrm{Q} \beta$ collected in the SRHA coadsorbate systems started to deviate from the respective adsorption profiles collected in the virus single-adsorbate systems (i.e., in the absence of SRHA). These deviations signify changes in the viscoelastic properties of the adlayers when adsorption transitioned from the first phases with high initial adsorption rates to the second phases with lower adsorption rates (see Figures $\mathbf{2 c , d}$ in the manuscript), consistent with adsorption of the GA and $\mathrm{Q} \beta$ onto the surfaces of the SRHA adlayers that formed on the $\mathrm{SAM}-\mathrm{NH}_{3}{ }^{+}$surfaces during the coadsorption process. 
a. MS2 + SRHA onto SAM- $-\mathrm{NH}_{3}{ }^{+}$surfaces

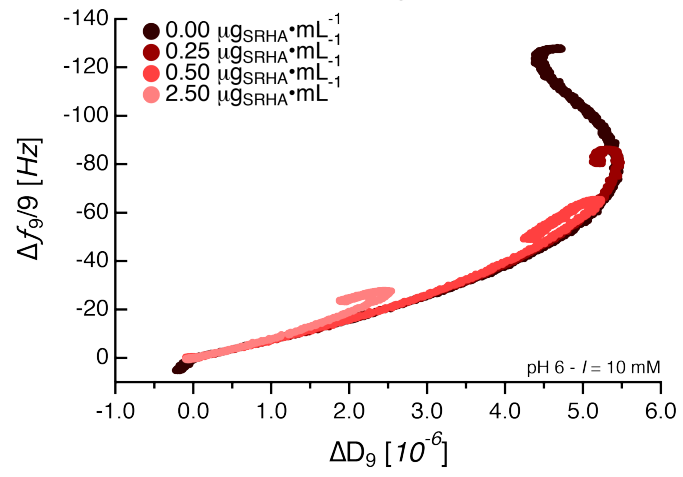

C. $\mathrm{GA}+\mathrm{SRHA}$ onto $\mathrm{SAM}^{-\mathrm{NH}_{3}+}$ surfaces

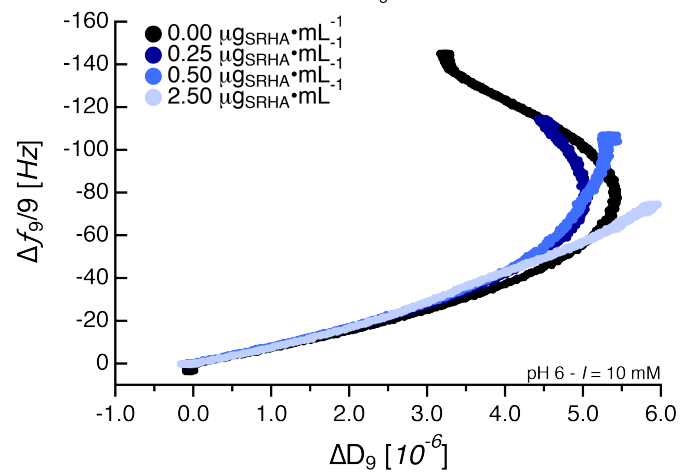

b. $\mathrm{fr}+\mathrm{SRHA}$ onto $\mathrm{SAM}-\mathrm{NH}_{3}{ }^{+}$surfaces

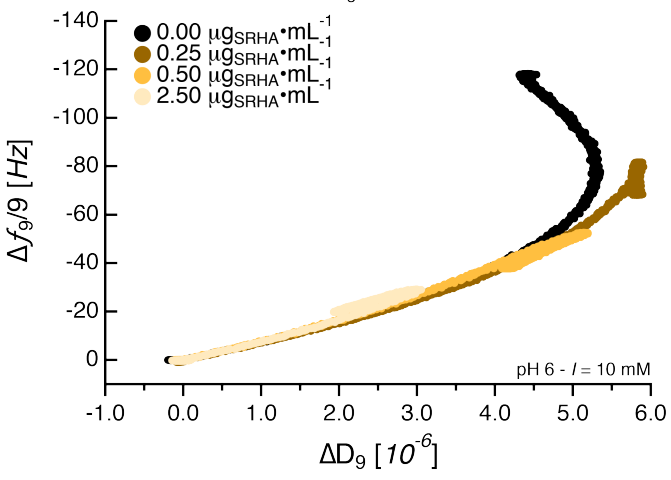

d. $\mathrm{Q} \beta+\mathrm{SRHA}$ onto SAM-NH${ }_{3}^{+}$surfaces

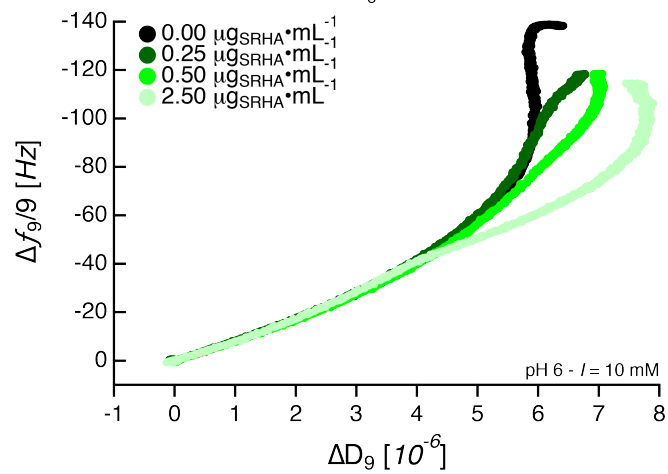

Figure S2. Dynamics in the viscoelastic properties of adlayers formed on the surfaces of amine terminated self-assembled monolayers $\left(\mathrm{SAM}_{\mathrm{NH}}{ }_{3}{ }^{+}\right.$) during adsorption of viruses (a) MS2, (b) fr, (c) GA, and (d) $\mathrm{Q} \beta$ in single-adsorbate systems (i.e., in the absence of Suwannee River humic acid (SRHA); $0.00 \mu \mathrm{g}_{\mathrm{SRHA}} \cdot \mathrm{mL}^{-1}$ ) and in the coadsorbate system with SRHA as the coadsorbate. The data is re-plotted from Figure 2a-d in the manuscript in the form of changes in the measured resonance frequency values of the ninth overtones $\left(\Delta f_{9} / 9\right)$ versus changes in the respective dissipation values $\left(\Delta D_{9}\right)$. Shown are representative adsorption profiles. All experiments were conducted at $\mathrm{pH} 6$ and at an ionic strength of $I=10 \mathrm{mM}$ using $\mathrm{NaCl}$ as the background electrolyte. The solution concentrations of SRHA in the coadsorbate systems were $0.25,0.50$, and $2.50 \mu \mathrm{g}_{\mathrm{SRHA}} \cdot \mathrm{mL}^{-1}$. 
S2.3 Adsorption of MS2 onto SAM-NH${ }_{3}^{+}$surfaces in the presence of the coadsorbate SRNOM

Figure $\mathbf{S 3}$ shows the changes in the total adsorbed masses on $\mathrm{SAM}-\mathrm{NH}_{3}{ }^{+}$ surfaces during MS2 adsorption in the absence of Suwannee River Natural Organic Matter (SRNOM) (i.e., single-adsorbate system) and in the presence of increasing concentrations of SRNOM as a coadsorbate. The SRNOM solution concentrations varied between 0.25 and $37.5 \mu \mathrm{g}_{\mathrm{SRNOM}} \cdot \mathrm{mL}^{-1}$. MS2 and SRNOM directly competed for adsorption sites on the $\mathrm{SAM}-\mathrm{NH}_{3}{ }^{+}$surfaces, as evidenced from decreasing final adsorbed masses (i.e., decreasing adsorbed masses of MS2) with increasing coadsorption of SRNOM.

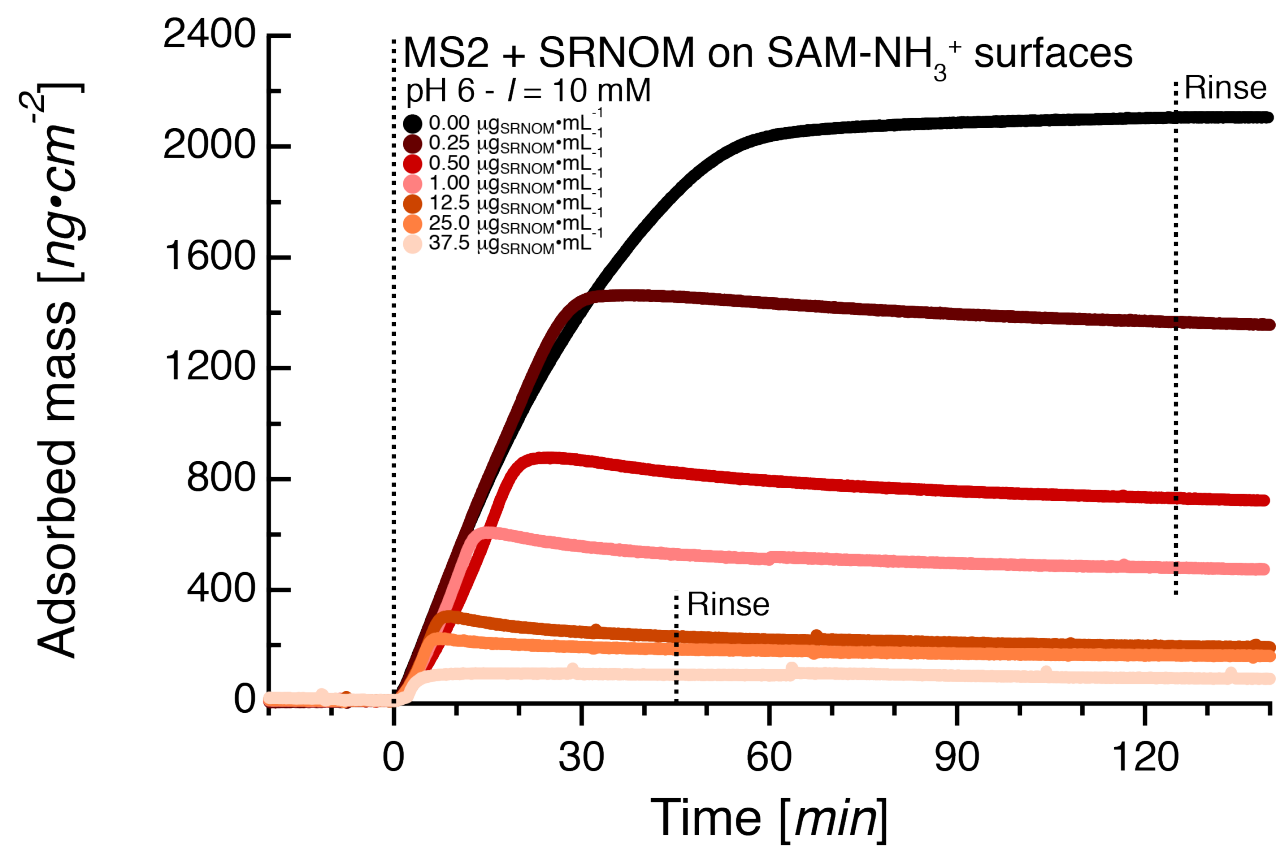

Figure S3. Changes in the adsorption profiles of MS2 onto amine-terminated self-assembled monolayer $\left(\mathrm{SAM}_{-} \mathrm{NH}_{3}{ }^{+}\right.$) surfaces during adsorption of MS2 in the absence of Suwannee River Natural Organic Matter (SRNOM) (i.e., single-adsorbate system) and in the presence of increasing concentrations of SRNOM as coadsorbate. All experiments were conducted at $\mathrm{pH} 6$ and at an ionic strength of $I=10 \mathrm{mM}$ (adjusted by $\mathrm{NaCl}$ ). The solution concentrations of SRNOM in the coadsorbate systems varied between 0.25 and $37.5 \mu \mathrm{g}_{\mathrm{SRNOM}} \cdot \mathrm{mL}^{-1}$. 
S2.4 Effects of solution ionic strength I on coadsorption of MS2 and SRHA onto SAM$\mathrm{NH}_{3}{ }^{+}$surfaces

In addition to the experiments conducted at a solution ionic strength of $I=10$ $\mathrm{mM}$, we studied adsorption dynamics in the MS2-SRHA coadsorbate systems at a higher ionic strength of $I=250 \mathrm{mM}$ (adjusted by $\mathrm{NaCl}$ ) while maintaining the same $\mathrm{pH}$ of 6. Figure S4a shows the adsorption profiles of SRHA onto $\mathrm{SAM}^{-\mathrm{NH}_{3}}{ }^{+}$ surfaces at $I=250 \mathrm{mM}$ and $\mathrm{pH} 6$ at the three tested SRHA concentrations of 0.25 , 0.50 , and $2.50 \mu \mathrm{g}_{\mathrm{SRHA}} \mathrm{mL}^{-1}$. As expected, the initial adsorption rates decreased with decreasing SRHA concentration. While the shapes of the SRHA adsorption profiles obtained at $I=250 \mathrm{mM}$ were similar to those obtained at $I=10 \mathrm{mM}$ (see Figure $1 \mathrm{c}$ in the manuscript), SRHA adsorption leveled off at higher final adsorbed masses at $I=$ $250 \mathrm{mM}$ (approximately $150-160 \mathrm{ng} \cdot \mathrm{cm}^{-2}$; irrespective of the SRHA concentration) than at $I=10 \mathrm{mM}$ (approximately $100 \mathrm{ng} \cdot \mathrm{cm}^{-2}$ ). The higher final adsorbed masses at the higher $I$ likely resulted from a higher conformational stability of the adsorbing SRHA due to charge screening of both intra-SRHA electrostatic repulsion and electrostatic attraction between SRHA and the $\mathrm{SAM}-\mathrm{NH}_{3}{ }^{+}$surfaces, leading to smaller extents of unfolding of the SRHA on the $\mathrm{SAM}_{-\mathrm{NH}_{3}}{ }^{+}$surfaces. $^{2}$

Figure S4b shows that the increase in the solution ionic strength from $I=10$ $\mathrm{mM}$ to $250 \mathrm{mM}$ resulted in increased adsorption of MS2 onto the SRHA adlayer. Adsorption of MS2 was slow yet continuous over the investigated timeframe. This increased adsorption was likely due to increased shielding of MS2-SRHA electrostatic repulsion at $I=250 \mathrm{mM}$ as compared to $I=10 \mathrm{mM}$. 
Figures S4c,d show the adsorption profiles and the corresponding final adsorbed masses of MS2 onto $\mathrm{SAM}-\mathrm{NH}_{3}{ }^{+}$surfaces in the absence of SRHA (i.e., single-adsorbate system) and of MS2 in the presence of varying concentrations of SRHA as coadsorbate. All experiments were run at $\mathrm{pH} 6$ and $I=250 \mathrm{mM}$. While the overall effects of SRHA on the MS2 adsorption profiles at $I=250 \mathrm{mM}$ were comparable to those at $I=10 \mathrm{mM}$ (comparison of Figure S4c,d and Figure 2a in the manuscript), adsorption plateaued at slightly higher final masses at $I=250 \mathrm{mM}$ than at $10 \mathrm{mM}$ (Figure S4d). The increased final adsorbed masses at the higher $I$ likely resulted from a number of factors. First, more effective screening of MS2-SRHA electrostatic repulsion at $I=250 \mathrm{mM}$ than $10 \mathrm{mM}$ resulted in slightly increased affinity of MS2 to the surfaces of SRHA adlayers (Figure S4b) that formed on the SAM-NH${ }_{3}{ }^{+}$surfaces during the coadsorption process. Second, charge screening at the high $I$ likely decreased the free area on the sorbent surface required for MS2 adsorption due to diminished MS2-MS2 and MS2-SRHA electrostatic repulsion in adsorbed states. Overall, the results show that increases in $I$ have a modulating effect on adsorption dynamics in the coadsorbate systems. The overall conclusions derived from the low $I$ coadsorbate experiments also hold true for the same systems at higher $I$. 
a. SRHA onto SAM- $\mathrm{NH}_{3}{ }^{+}$surfaces

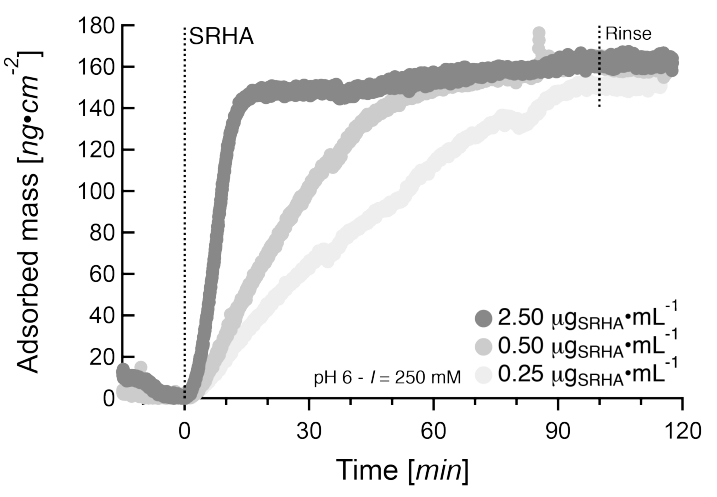

C. MS2 + SRHA onto SAM- $\mathrm{NH}_{3}{ }^{+}$surfaces

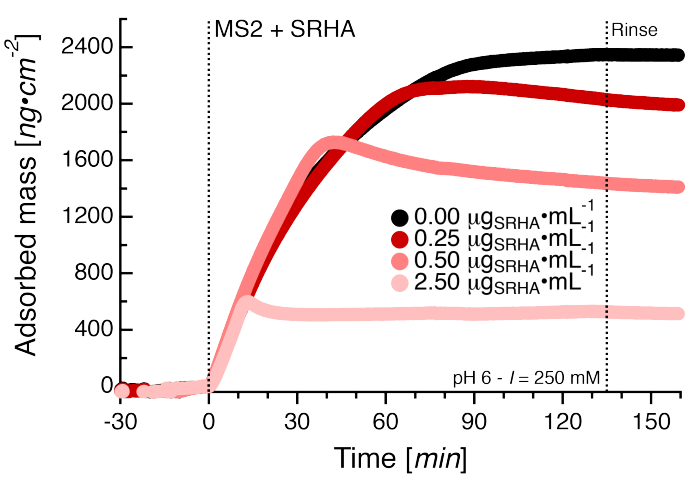

b. MS2 onto SRHA adlayers

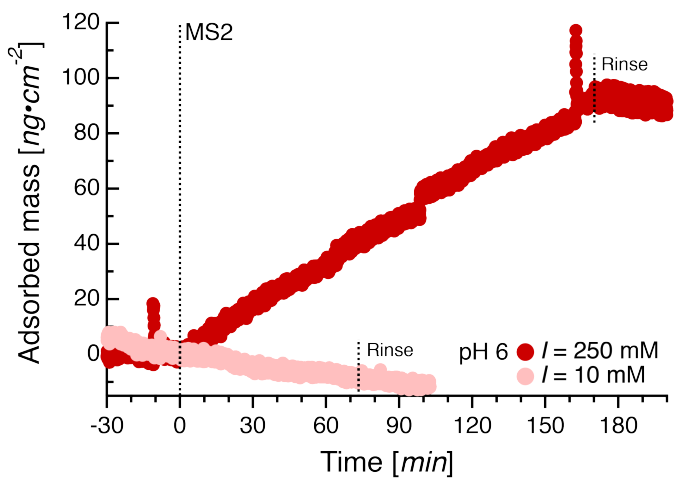

d. $\mathrm{MS} 2+\mathrm{SRHA}$ onto $\mathrm{SAM}^{-\mathrm{NH}_{3}}$ : final adsorbed masses
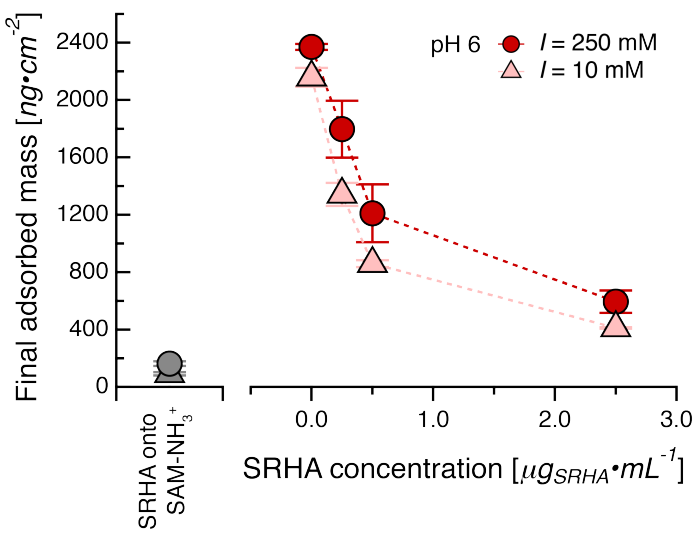

Figure S4. Effects of solution ionic strength $I$ on the adsorption of MS2 and Suwannee River humic acid (SRHA) onto amine-terminated self-assembled monolayer ( $\mathrm{SAM}-\mathrm{NH}_{3}{ }^{+}$) surfaces in MS2 singleadsorbate and virus-SRHA coadsorbate systems. (a) Adsorption profiles of SRHA onto SAM- $\mathrm{NH}_{3}{ }^{+}$ surfaces at varying SRHA concentrations. All experiments were conducted at $\mathrm{pH} 6$ and $I=250 \mathrm{mM}$ (adjusted by $\mathrm{NaCl}$ ). (b) Adsorption profiles of MS2 onto SRHA adlayers during initial phases of adsorption at $I=250 \mathrm{mM}$ and $10 \mathrm{mM}$ (drawn for comparison). Both adsorption experiments were run at $\mathrm{pH} 6$ and the $I$ was adjusted by $\mathrm{NaCl}$. The shown data is re-plotted from Armanious et al. (2016). ${ }^{1}$ (c) Adsorption profiles of MS2 onto SAM- $\mathrm{NH}_{3}{ }^{+}$surfaces in the absence of SRHA (i.e., MS2 singleadsorbate system) and in the presence of varying concentrations of SRHA as coadsorbate. All experiments were conducted at pH 6 and ionic strength $I=250 \mathrm{mM}$ (adjusted by $\mathrm{NaCl}$ ). (d) Final adsorbed masses at which adsorption plateaued for adsorption of MS2 in the absence and in the presence of varying concentrations of SRHA as coadsorbate. The values and error bars correspond to averages and ranges of duplicate measurements. All experiments were conducted at $\mathrm{pH} 6$ and either at $I=10 \mathrm{mM}$ (triangles) or $250 \mathrm{mM}$ (circles) (both adjusted with $\mathrm{NaCl}$ ). The grey symbols represent the final adsorbed masses of SRHA adlayers on the SAM- $\mathrm{NH}_{3}{ }^{+}$surfaces at $I=10 \mathrm{mM}$ (triangles) and $I=$ $250 \mathrm{mM}$ (circles). 


\section{S2.5 Adsorption of MS2 onto SAM- $\mathrm{NH}_{3}{ }^{+}$surfaces pre-coated with SRHA}

Figure S5a shows three adsorption profiles of SRHA obtained by delivering the same SRHA solution (concentration of $0.25 \mu \mathrm{g}_{\mathrm{SRHA}} \cdot \mathrm{mL}^{-1}$ ) to separate flow cells containing sensors with $\mathrm{SAM}-\mathrm{NH}_{3}{ }^{+}$surfaces for 15,30 , and 45 minutes before starting to rinse with a SRHA-free solution of the same $\mathrm{pH}$ and $I$. The final adsorbed SRHA masses, and hence the extents to which the $\mathrm{SAM}-\mathrm{NH}_{3}{ }^{+}$surfaces were coated by SRHA, increased with increasing the time span over which the SRHA solution was delivered to the flow cells. The fourth "adsorption profile" (labeled " 0 min") was obtained in a forth flow cell to which a SRHA-free solution was continuously delivered. There was no adsorption onto the $\mathrm{SAM}-\mathrm{NH}_{3}{ }^{+}$surface in this cell such that the changes in the mass over time are a measure of the instrument drift. Figure S5b,c show the adsorption profiles of MS2 and the corresponding final adsorbed masses on the sensors pre-coated with SRHA adlayers. Increasing amounts of SRHA preadsorbed onto the $\mathrm{SAM}-\mathrm{NH}_{3}{ }^{+}$surfaces resulted in decreasing initial adsorption rates and final adsorbed masses of MS2. This trend confirms that MS2 and SRHA directly competed for adsorption sites on the $\mathrm{SAM}-\mathrm{NH}_{3}{ }^{+}$surfaces and that there was very little, if any, adsorption of MS2 onto the SRHA adlayers. 

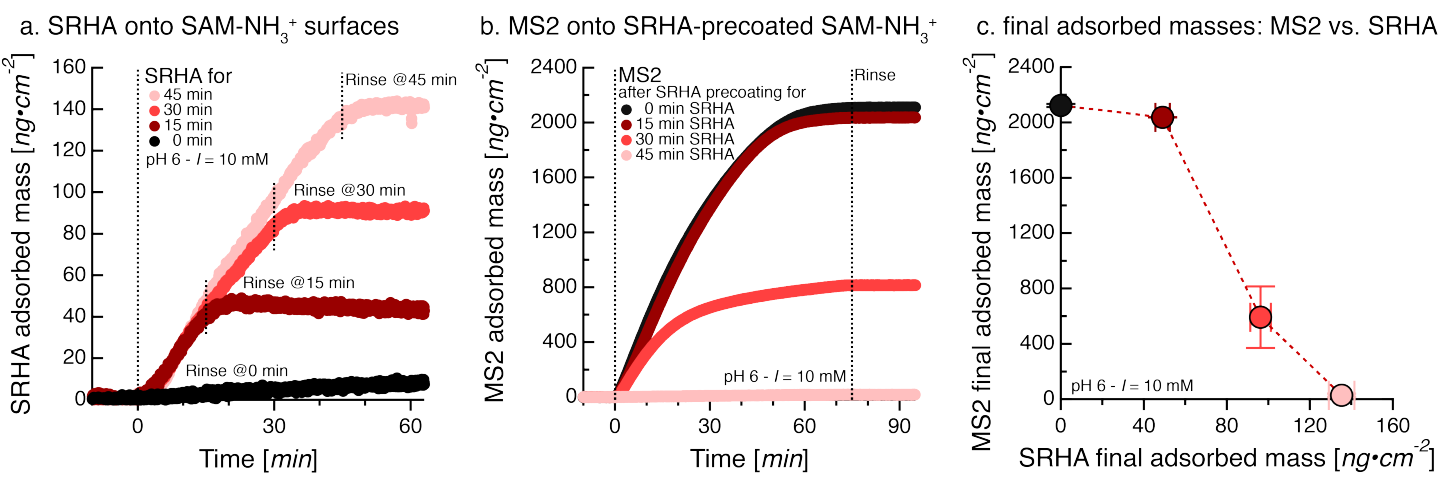

Figure S5. Adsorption of MS2 onto amine terminated self-assembled monolayer $\left(\mathrm{SAM}-\mathrm{NH}_{3}{ }^{+}\right)$surfaces pre-coated to different extents with Suwannee River humic acid (SRHA). (a) Adsorption profiles of SRHA onto SAM- $\mathrm{NH}_{3}{ }^{+}$surfaces obtained by delivering a SRHA-containing solution (concentration of $\left.0.25 \mu \mathrm{g}_{\mathrm{SRHA}} \cdot \mathrm{mL}^{-1}\right)$ for different periods of time $(15,30$, and 45 minutes) to the sensor surfaces in three separate flow cells, followed by rinsing the cells with a SRHA-free solution. For reference purposes, a SRHA-free solution was continuously delivered to a fourth flow cell containing a sensor with a SAM$\mathrm{NH}_{3}{ }^{+}$surface. The change in mass over time in this flow cell (i.e., labeled " 0 min") is a direct measure of the instrument drift. (b) Adsorption of MS2 onto $\mathrm{SAM}-\mathrm{NH}_{3}{ }^{+}$surfaces pre-coated with different amounts of SRHA, as shown in panel (a). (c) Average final adsorbed masses of MS2 on SAM- $\mathrm{NH}_{3}{ }^{+}$ surfaces as a function of the amounts of SRHA that were pre-coated onto the SAM-NH${ }_{3}^{+}$surfaces prior to MS2 adsorption. The values and error bars correspond to the averages and ranges of duplicate measurements, respectively. All experiments were conducted at $\mathrm{pH} 6$ and $I=10 \mathrm{mM}$ (adjusted by $\mathrm{NaCl}$ ) and with a constant volumetric flow rate of $20 \mu \mathrm{L} \cdot \mathrm{min}^{-1}$.

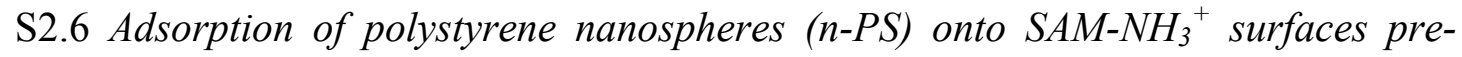
coated with SRHA.

Figure S6a shows three adsorption profiles of SRHA obtained by delivering the same SRHA solution (concentration of $0.25 \mu \mathrm{g}_{\mathrm{SRHA}} \cdot \mathrm{mL}^{-1}$ ) to three separate flow cells containing sensors with $\mathrm{SAM}-\mathrm{NH}_{3}{ }^{+}$surfaces for 20,40 , and 60 minutes before starting to rinse the surfaces with a SRHA-free solution of the same $\mathrm{pH}$ and $I$. The final adsorbed SRHA masses, and hence the extents to which the $\mathrm{SAM}-\mathrm{NH}_{3}{ }^{+}$surfaces were coated by SRHA, increased with increasing the time span over which the SRHA solution was delivered to the flow cells. The fourth "adsorption profile" (labeled "0 min") was obtained in a forth flow cell to which a SRHA-free solution was continuously delivered. There was no adsorption to the $\mathrm{SAM}-\mathrm{NH}_{3}{ }^{+}$surface in this cell 
such that the changes in the mass over time are a measure of the instrument drift.

Figure S6b,c show the adsorption profiles of n-PS and the corresponding final adsorbed masses to the sensors pre-coated with SRHA adlayers. Increasing amounts of SRHA pre-adsorbed onto the $\mathrm{SAM}-\mathrm{NH}_{3}{ }^{+}$surfaces resulted in decreasing initial adsorption rates and final adsorbed masses of n-PS. This trend confirms that n-PS and SRHA directly competed for adsorption sites on the $\mathrm{SAM}-\mathrm{NH}_{3}{ }^{+}$surfaces and that that there was very little, if any, adsorption of n-PS onto the SRHA adlayers.
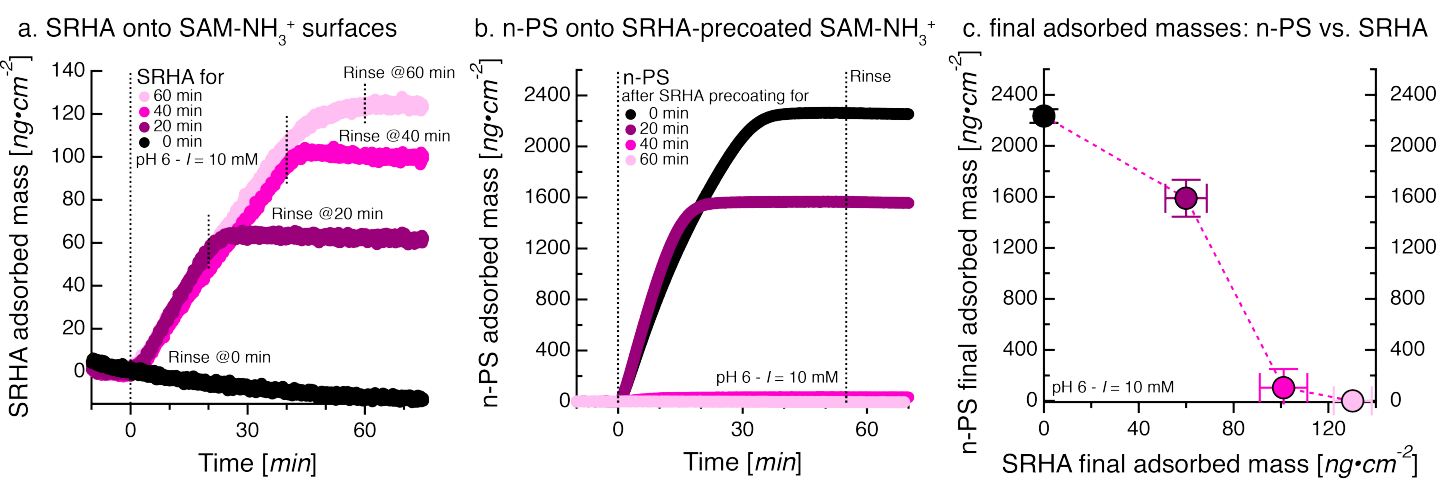

Figure S6. Adsorption of the polystyrene nanospheres (n-PS) onto amine terminated self-assembled monolayer $\left(\mathrm{SAM}-\mathrm{NH}_{3}{ }^{+}\right.$) surfaces pre-coated to different extents with Suwannee River humic acid (SRHA). (a) Adsorption profiles of SRHA onto $\mathrm{SAM}^{-\mathrm{NH}_{3}}{ }^{+}$surfaces obtained by delivering a SRHAcontaining solution (concentration of $0.25 \mu \mathrm{g}_{\mathrm{SRHA}} \cdot \mathrm{mL}^{-1}$ ) for different periods of time $(20,40$, and 60 minutes) to the sensor surfaces in three separate flow cells, followed by rinsing the cells with a SRHAfree solution. For reference purposes, a SRHA-free solution was continuously delivered to a fourth flow cell containing a sensor with a $\mathrm{SAM}-\mathrm{NH}_{3}{ }^{+}$surface. The change in mass over time in this flow cell (i.e., labeled " 0 min") is a direct measure of the instrument drift. (b) Adsorption of n-PS onto SAM$\mathrm{NH}_{3}{ }^{+}$surfaces pre-coated with different amounts of SRHA, as shown in panel (a). (c) Average final adsorbed masses of n-PS as a function of the amounts of SRHA that were pre-coated onto the SAM$\mathrm{NH}_{3}{ }^{+}$surfaces prior to adsorption of n-PS. The values and error bars correspond to the averages and ranges of duplicate measurements, respectively. All experiments were conducted at $\mathrm{pH} 6$ and $I=10$ $\mathrm{mM}$ (adjusted by $\mathrm{NaCl}$ ) and with a constant volumetric flow rate of $20 \mu \mathrm{L} \cdot \mathrm{min}^{-1}$. 
S2.7 Predicting adsorbed masses in MS2-SRHA coadsorbate systems based on singleadsorbate adsorption profiles of MS2 and SRHA.

Figure S7 show the adsorption profiles of MS2 (panel a) and SRHA (panel b) onto $\mathrm{SAM}-\mathrm{NH}_{3}{ }^{+}$surfaces obtained in the single-adsorbate systems. The data is replotted from Figures 1a and 1c as fractions of the final adsorbed masses versus time. Based on the simplifying assumption that the fractional mass linearly correlates with the fractional coverage of the $\mathrm{SAM}-\mathrm{NH}_{3}{ }^{+}$surfaces, it is possible to estimate the times needed to reach full surface coverages (i.e., the jamming limits of the $\mathrm{SAM}-\mathrm{NH}_{3}{ }^{+}$ surfaces) in the MS2-SRHA coadsorbate systems by adding the fractional masses of the MS2 and the SRHA single-adsorbate adsorption profiles. Using this approach, we estimated the times at which the jamming limits are reached in the three tested MS2SRHA coadsorbate systems (Figure S7c). As expected, the times decrease with increasing concentration of SRHA as coadsorbate (i.e., approximately 22, 17, and 8 minutes for concentrations of $0.25,0.50$, and $2.50 \mu \mathrm{g}_{\mathrm{SRHA}} \cdot \mathrm{mL}^{-1}$, respectively).

Figure S7d shows the adsorption profiles of MS2 onto $\mathrm{SAM}_{-\mathrm{NH}_{3}}{ }^{+}$surface (re-plotted from Figure 2a in the manuscript) in the absence of SRHA (single adsorbate system) and in the presence of SRHA at the three tested concentrations. We note that the same SRHA concentrations were used in the SRHA single-adsorbate systems (Figure 1c in the manuscript and Figure 7b) and in the MS2-SRHA coadsorbate systems (Figure 2a in the manuscript and Figure S7d). The grey vertical arrows correspond to the estimated times that were required to reach the jamming limits for MS2 and SRHA on the $\mathrm{SAM}-\mathrm{NH}_{3}{ }^{+}$surfaces in the coadsorbate systems. We 
approximated the adsorbed masses of MS2 at these times from the MS2 singleadsorbate adsorption profile (labeled " $0 \mu \mathrm{g}_{\mathrm{SRHA}} \cdot \mathrm{mL}^{-1}$ " in Figure S7d). The rationale behind this approximation was that MS2 is much larger and adsorbed at much higher initial rates than SRHA and hence is expected to have much larger contributions than SRHA to the final adsorbed masses in the coadsorbate systems.

Figure S7d shows a very good agreement between the predicted and the experimental final adsorbed masses in the coadsorbate systems with 0.25 and 0.50 $\mu \mathrm{g}_{\mathrm{SRHA}} \cdot \mathrm{mL}^{-1}$. However, the predicted final adsorbed mass was much smaller than the experimental values at the highest SRHA concentration of $2.50 \mu \mathrm{g}_{\mathrm{SRHA}} \cdot \mathrm{mL}^{-1}$ (i.e., a predicted mass of approximately $1050 \mathrm{ng} \cdot \mathrm{cm}^{-2}$ instead of an experimental mass of $1400 \mathrm{ng} \cdot \mathrm{cm}^{-2}$ ). It is possible that the discrepancy between the predicted and measured final masses resulted from the fact that this simple approach does not account for changes in mass contributions of water to the measured total mass as a function of surface coverage and the simplifying assumption that fractional masses measured by QCM-D correlate linearly with fractional coverages of the sensors. We thus decided to develop a more sophisticated random sequential adsorption model to obtain a better understanding of the adsorption dynamics in the MS2-SRHA coadsorbate systems. 

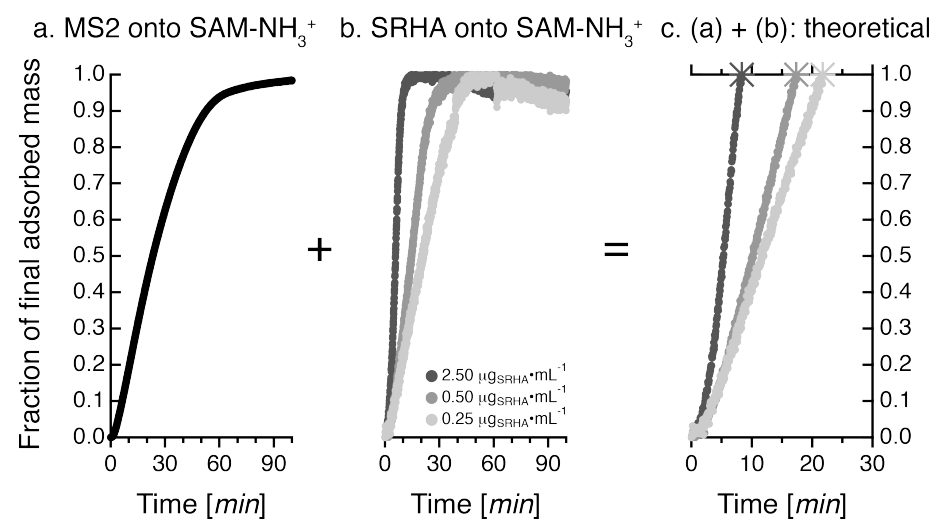

d. $(a)+(b)$ : experimental

Figure S7. (a) Adsorption profile of MS2 onto an amine-terminated self-assembled monolayer (SAM$\mathrm{NH}_{3}{ }^{+}$) surface in the absence of Suwannee River humic acid (SRHA), re-plotted from Figure 2a. The changes in adsorbed mass are plotted as fractions of the final adsorbed mass. (b) Adsorption profiles of SRHA onto SAM- $\mathrm{NH}_{3}{ }^{+}$surfaces as a function of the SRHA concentration, re-plotted from Figure 1c. The changes in adsorbed mass are plotted as fractions of the final adsorbed mass. (c) Predicted changes in the adsorbed masses over time in the three coadsorbate systems by time-wise addition of the singleadsorbate adsorption profiles of MS2 (panel a) and SRHA (panel b). (d) Comparison of predicted and experimental final adsorbed masses in the MS2-SRHA coadsorbate systems at varying SRHA concentrations.

\section{S2.8 Modeling coadsorption systems of MS2 and SRHA using an RSA Monte Carlo}

model

Dimensions of MS2 virions and SRHA assemblies. To model the coadsorbate systems, we first converted the initial adsorption rates of MS2 and SRHA onto SAM$\mathrm{NH}_{3}{ }^{+}$surfaces measured in the single-adsorbate systems to absolute numbers of MS2 virions and SRHA 'assemblies' that adsorbed per time increment. Absolute numbers of the two coadsorbates were required for the subsequent modeling of competition. More specifically, these numbers were required to accurately model the number of SRHA 'assemblies' that approached the $\mathrm{SAM}^{-\mathrm{NH}_{3}}{ }^{+}$surface per MS2 virion that approached the surface. Increases in the number of SRHA assemblies that coadsorb with each MS2 virion are expected to increase competitive inhibition of MS2 adsorption. 
Based on its crystallographic structure, ${ }^{5} \mathrm{MS} 2$ was modeled as a sphere with a diameter of $28.8 \mathrm{~nm}$ (Table 1 in the manuscript). ${ }^{1}$ SRHA assemblies were modeled using two different conceptual approaches. In the first approach, SRHA assemblies were considered to have spherical shapes in solution and a high structural stability. Because of this stability, it was assumed that SRHA maintained a spherical conformation when adsorbing from solution onto the $\mathrm{SAM}-\mathrm{NH}_{3}{ }^{+}$surfaces. In this case, the diameter of the spherical SRHA assemblies could be approximated by the thickness of the SRHA adlayers forming on the $\mathrm{SAM}-\mathrm{NH}_{3}{ }^{+}$surfaces. The final adsorbed masses of SRHA on $\mathrm{SAM}_{-} \mathrm{NH}_{3}{ }^{+}$at $I=10 \mathrm{mM}$ were approximately $\approx 100$ $\mathrm{ng} \cdot \mathrm{cm}^{-2}$ (see Figure 1c in the manuscript). Using published SRHA adlayer density values of $1.05 \mathrm{~g} \cdot \mathrm{cm}^{-3}$, 2,6-8 the SRHA adlayer thickness - and thus the diameter of spherical SRHA assemblies in solution — was approximately $1.0 \mathrm{~nm}$.

The second approach also assumed that SRHA assemblies have a spherical shape in solution, but - in contrast to the first approach - a low structural stability. As a consequence, it was assumed that the SRHA unfolded on the $\mathrm{SAM}-\mathrm{NH}_{3}{ }^{+}$ surfaces upon adsorption, thereby adapting flatter, disk-shaped conformations in adsorbed states. The measured final adsorbed masses at $I=10 \mathrm{mM}$ and $\mathrm{pH} 6$ of $\approx 100$ $\mathrm{ng} \cdot \mathrm{cm}^{-2}$ thus reflected the height of the unfolded SRHA assemblies but not the diameters of the SRHA assemblies in solution prior to adsorption. The height of the unfolded SRHA assemblies was approximately $\approx 1.0 \mathrm{~nm}$, as detailed above. 
The diameter of the unfolded SRHA assemblies in solution is not easily accessible by direct measurements. We therefore estimated the diameter by measuring the thicknesses of SRHA adlayers formed on $\mathrm{SAM}_{-\mathrm{NH}_{3}}{ }^{+}$surfaces at $I=500 \mathrm{mM}$ and pH 6 and a high SRHA concentration of $50 \mu \mathrm{g}_{\mathrm{SRHA}} \cdot \mathrm{mL}^{-1}$. We used the high ionic strength and a high concentration of SRHA to decrease (or ideally to completely impair) SRHA unfolding upon adsorption. First, increasing $I$ is expected to increase the structural stability of SRHA due to increased charge screening of both intraSRHA electrostatic repulsion and electrostatic attraction between SRHA and the SAM- $-\mathrm{NH}_{3}{ }^{+}$surfaces. ${ }^{2}$ Increasing structural stability of polyelectrolytes in adsorbed states with increasing $I$ is well documented in the literature. ${ }^{9,10}$ Second, the high SRHA concentration resulted in rapid filling of the $\mathrm{SAM}-\mathrm{NH}_{3}{ }^{+}$surface and hence decreased the time available for individual SRHA assemblies to unfold. Impaired unfolding by rapid filling of adsorbent surfaces is well documents for structurally instable (i.e., 'soft') proteins. ${ }^{11-14}$

The final adsorbed masses of SRHA on $\mathrm{SAM}_{-} \mathrm{NH}_{3}{ }^{+}$surfaces at $I=500 \mathrm{mM}$ was $\approx 290 \mathrm{ng} \cdot \mathrm{cm}^{-2}$ (Figure S8a). Based on a SRHA adlayer density of $1.05 \mathrm{~g} \cdot \mathrm{cm}^{-3}, 2,6-$ ${ }^{8}$ the estimated diameter of a spherical SRHA 'molecule' in solution is $\approx 2.7 \mathrm{~nm}$ at $I=$ $10 \mathrm{mM}$.

Evidence for unfolding of SRHA assemblies upon adsorption. Figure S8a shows adsorption profiles of SRHA at a constant concentration of $50.0 \mu \mathrm{g} \cdot \mathrm{mL}^{-1}$ at constant $\mathrm{pH} 6$ but at varying ionic strengths of $I=10$ to $500 \mathrm{mM}$ (adjusted by $\mathrm{NaCl}$ ). 
Increasing $I$ is considered to increase the structural stability of SRHA assemblies by screening intra-SRHA electrostatic repulsion and electrostatic attraction between SRHA and the $\mathrm{SAM}-\mathrm{NH}_{3}{ }^{+}$surfaces. Decreased unfolding of polyelectrolytes on adsorbent surfaces with increasing $I$ is well established and has been ascribed to the polyelectrolytes adopting more condensed, coil-like structures at high $I .^{9,10}$ The adsorption profiles at different $I$ all show comparable initial adsorption rates, suggesting that a comparable number of similarly sized SRHA assemblies attached to the $\mathrm{SAM}-\mathrm{NH}_{3}{ }^{+}$surfaces per unit time. The final mass at which SRHA adsorption plateaued, however, increased with increasing $I$. This trend suggests that decreasing $I$ increased the rate at which adsorbed SRHA assemblies unfolded into flatter conformation on the sorbent surface, thereby increasing the area on the sorbent surface that is occupied by each SRHA assembly. We note that a higher SRHA concentration of $50 \mu \mathrm{g}_{\mathrm{SRHA}} \cdot \mathrm{mL}^{-1}$ was used than in the experiments shown in Figure S4 (concentration up to $2.50 \mu \mathrm{g}_{\mathrm{SRHA}} \cdot \mathrm{mL}^{-1}$ ). The higher SRHA concentration resulted in thicker SRHA adlayers, as discussed in detail below.

Figure S8b shows the adsorption profiles of SRHA to $\mathrm{SAM}-\mathrm{NH}_{3}{ }^{+}$surfaces at different solution concentrations of SRHA. All experiments were conducted at $\mathrm{pH} 6$ and $I=10 \mathrm{mM}$. Adsorption profiles collected at SRHA concentrations between 0.25 and $2.50 \mu \mathrm{g}_{\mathrm{SRHA}} \cdot \mathrm{mL}^{-1}$ plateaued at comparable final adsorbed masses of approximately $100 \mathrm{ng} \cdot \mathrm{cm}^{-2}$, adsorption at $50.0 \mu \mathrm{g}_{\mathrm{SRHA}} \cdot \mathrm{mL}^{-1}$ plateaued at a substantially higher final adsorbed mass of approximately $140 \mathrm{ng} \cdot \mathrm{cm}^{-2}$. The increase in final adsorbed mass with increasing SRHA concentration provides indirect 
evidence for unfolding dynamics of SRHA assemblies on the $\mathrm{SAM}-\mathrm{NH}_{3}{ }^{+}$surfaces upon adsorption. At the highest tested SRHA concentration of $50 \mu \mathrm{g}_{\mathrm{SRHA}} \cdot \mathrm{mL}^{-1}$, SRHA assemblies were delivered to the surface at a comparatively high rate. As a consequence, the adsorbent surface was rapidly covered by SRHA assemblies and attained densely packed states at a rate that was comparable or even faster than the rate by which adsorbed SRHA assemblies unfolded. In other words, before adsorbed SRHA assemblies could unfold, the space around them required for the unfolding process was already extensively occupied by other adsorbed SRHA assemblies. At the three lower SRHA concentrations of $0.25,0.50$, and $2.50 \mu \mathrm{g}_{\mathrm{SRHA}} \cdot \mathrm{mL}^{-1}$, sufficient time was available for adsorbed SRHA assemblies to unfold before the area surrounding the adsorbed SRHA became occupied by additional SRHA assemblies adsorbing from solution. Sufficient time for complete unfolding is a plausible explanation why adsorption at the three lower SRHA concentrations of $0.25,0.50$, and 2.50 $\mu \mathrm{g}_{\mathrm{SRHA}} \cdot \mathrm{mL}^{-1}$ resulted in comparable final adsorbed masses. The pronounced increase in final adsorbed SRHA mass from 2.50 to $50 \mu \mathrm{g}_{\mathrm{SRHA}} \cdot \mathrm{mL}^{-1}$ suggests that the unfolding process was relatively fast and was completed within a few minute of adsorption.

In combination, the data shown in Figure $\mathbf{S 8}$ provides strong evidence for unfolding of SRHA upon adsorption to oppositely charged surfaces. This process is well documented for proteins and polyelectrolytes. ${ }^{9-14}$ We used the thickness of SRHA adlayers formed on $\mathrm{SAM}^{-\mathrm{NH}_{3}}{ }^{+}$surfaces at $\mathrm{pH} 6$ and $I=500 \mathrm{mM}$ and at a concentration of $50 \mu \mathrm{g}_{\mathrm{SRHA}} \cdot \mathrm{mL}^{-1}$ to estimate the diameters of SRHA in solution and 
upon adsorption, because SRHA is expected to be the most stable at the highest tested $I$.

a. SRHA onto SAM- $\mathrm{NH}_{3}{ }^{+}$surfaces at different $I$

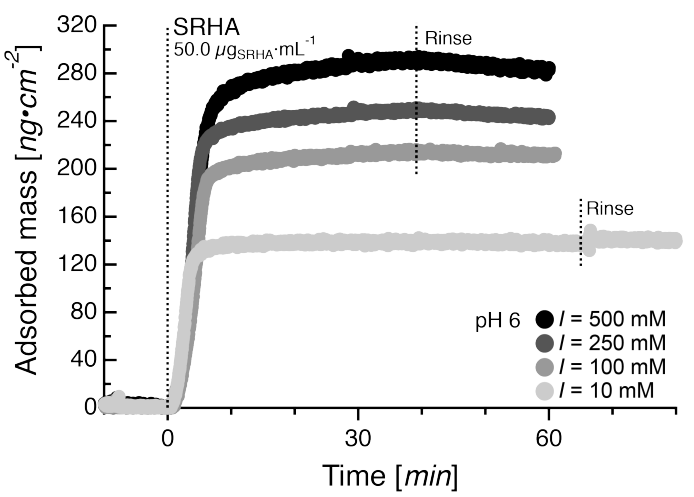

b. SRHA onto SAM- $\mathrm{NH}_{3}{ }^{+}$surfaces at different conc.

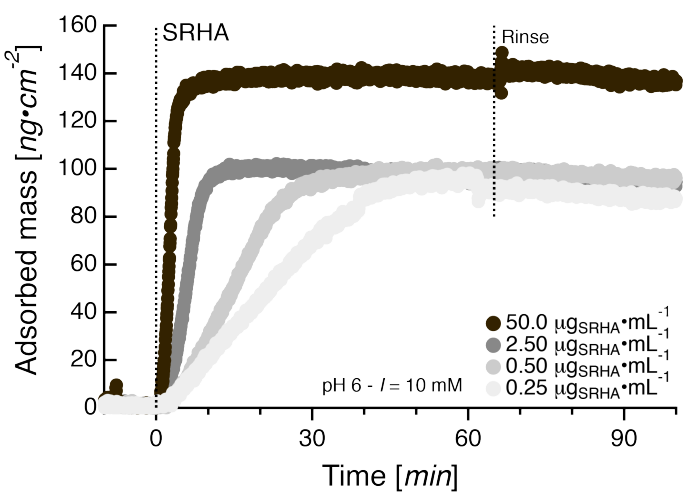

Figure S8. Adsorption profiles of Suwannee River humic acid (SRHA) to amine-terminated selfassembled monolayers (SAM- $\mathrm{NH}_{3}{ }^{+}$) surfaces. (a) Effects of variations in the ionic strength at different ionic strengths $I$ from 10 to $500 \mathrm{mM}$ (adjusted by $\mathrm{NaCl}$ ) at a constant SRHA concentration of 50 $\mu \mathrm{g} \cdot \mathrm{mL}^{-1}$ and $\mathrm{pH}$ 6. (b) Effects of variations in the SRHA concentrations at constant solution ionic strength $I=10 \mathrm{mM}$ and $\mathrm{pH} 6$.

Determining the hydrated wet mass of individual MS2 virions and SRHA assemblies. We subsequently used the estimated SRHA diameters in solution, $\mathrm{d}_{\mathrm{SRHA}}^{\text {solution }}$, to convert the initial adsorption rates measured in the MS2 and SRHA single-adsorbate systems to number ratios of MS2 virions and SRHA assemblies that adsorbed per unit time. For the interpretation of the initial adsorption rates measured by QCM-D, it is important to note that this technique senses not only the 'dry' mass of adsorbates but also the mass of water that hydrodynamically couples with the adsorbates to the oscillation of the QCM-D sensor. ${ }^{15}$ The QCM-D measured adsorbed mass thus corresponds to a 'wet' adlayer mass. We used a hydration model of a truncated pyramid (Figure S9), proposed by Bingen et al $(2008)^{16}$, to estimate the 'wet' masses of each MS2 virion and SRHA assembly that adsorbed onto the SAM$\mathrm{NH}_{3}{ }^{+}$surfaces according to: 
$M_{\text {hydrated }}=\rho_{\text {water }} * V_{\text {pyramid }}+\left(\rho_{\text {MS2 or SRHA }}-\rho_{\text {water }}\right) * V_{\text {MS2 or SRHA sphere }}$

Eq. S1

where $M_{\text {hydrated }}(\mathrm{kg})$ is the 'wet' mass of an adsorbate (i.e., of a single MS2 virion or a single SRHA assembly) including the hydration shell, $\rho_{\text {water }}$ is the density of water $\left(=1000 \mathrm{~kg} \cdot \mathrm{m}^{-3}\right), V_{\text {pyramid }}\left(\mathrm{m}^{3}\right)$ is the volume of the truncated hydration pyramid, $\rho_{\mathrm{MS} 2}$ or SRHA $\left(\mathrm{kg} \cdot \mathrm{m}^{-3}\right)$ is the density of a MS2 virion or a SRHA assembly, and $V_{\text {MS2 or SRHA sphere }}\left(\mathrm{m}^{3}\right)$ is the volume of the spherical MS2 or SRHA assembly.

a. MS2

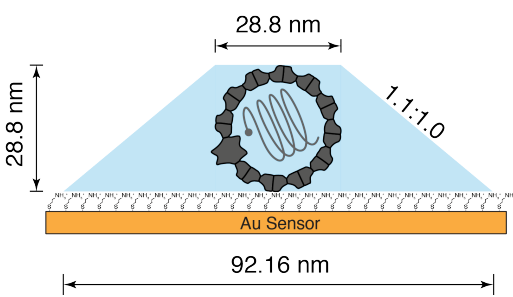

b. SRHA at $\mathrm{pH} 6 \& I=10 \mathrm{mM}$

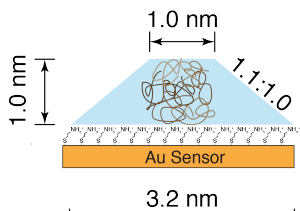

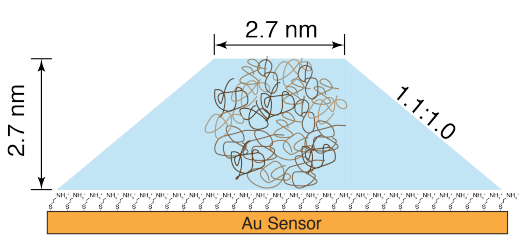

$8.64 \mathrm{~nm}$

Figure S9. The dimensions and shapes of the hydration shells of (a) MS2 and (b) and (c) Suwannee River humic acid (SRHA). The SRHA sphere shown in panel $b$ corresponds to the first conceptual approach in which we assumed that SRHA assemblies are structurally stable and do not unfold on the sorbent surface. The experimentally measured SRHA adlayer thickness are then good estimates of the diameter of spherical SRHA assemblies in solution. The SRHA sphere shown in panel c corresponds to the second conceptual approach in which we assumed that SRHA assemblies have a low structural stability and hence unfold on the adsorbent surface, adapting a flatter disk shaped conformation in adsorbed states than in solution. Panel c shows the dimensions of the SRHA sphere prior to unfolding. The schematic was adopted from Bingen et al $(2008)^{16}$.

Table $\mathbf{S 2}$ provides an overview of the estimated values of $V_{\text {pyramid }}, V_{\text {sphere, }}$, $\rho_{\text {MS2 or SRHA }}$, and $M_{\text {hydrated }}$ for MS2 and SRHA. The table contains two entries for SRHA corresponding to the two estimated $\mathrm{d}_{\mathrm{SRHA}}^{\text {solution }}$. 
Table S2. Dimensions and hydrated masses, $M_{\text {hydrated }}$, of MS2 virions and Suwannee River humic acid (SRHA) assemblies as well as their densities, $\rho_{\mathrm{MS} 2}$ or SRHA, and volumes of the hydration pyramids, $V_{\text {pyramid. }}$

\begin{tabular}{|c|c|c|c|c|c|}
\hline adsorbate & $\begin{array}{l}\text { diameter } \\
\mathrm{nm}\end{array}$ & $\begin{array}{l}\boldsymbol{V}_{\text {pyramid }} \\
\mathrm{m}^{3}\end{array}$ & $\begin{array}{l}\boldsymbol{V}_{\text {sphere }} \\
\mathrm{m}^{3}\end{array}$ & $\begin{array}{l}\boldsymbol{\rho}_{\text {MS } 2} \text { or SRHA } \\
\mathrm{kg} \cdot \mathrm{m}^{-3}\end{array}$ & $\begin{array}{l}\boldsymbol{M}_{\text {hydrated }} \\
\mathrm{kg}\end{array}$ \\
\hline $\mathrm{MS} 2$ & 28.8 & $1.15 \cdot 10^{-22}$ & $1.25 \cdot 10^{-23}$ & $1420^{\mathrm{a}}$ & $1.20 \cdot 10^{-19}$ \\
\hline $\mathrm{SRHA}_{\mathrm{pH} 6-I=10 \mathrm{mM}}$ & 1.0 & $4.81 \cdot 10^{-27}$ & $5.24 \cdot 10^{-28}$ & $1050^{\mathrm{b}}$ & $4.82 \cdot 10^{-24}$ \\
\hline 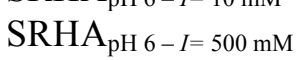 & 2.7 & $9.47 \cdot 10^{-26}$ & $1.03 \cdot 10^{-26}$ & $1050^{\mathrm{b}}$ & $9.53 \cdot 10^{-23}$ \\
\hline
\end{tabular}

Converting initial MS2 and SRHA adsorption rates to absolute numbers of MS2 virions and SRHA 'assemblies' adsorbing per time. We used the estimated wet masses, $M_{\text {hydrated }}$, of each MS2 virion and SRHA assembly and the measured initial adsorption rates of MS2 and SRHA from the single-adsorbate experiments to determine the absolute number of individual MS2 virions and of individual SRHA assemblies that adsorbed onto the $\mathrm{SAM}-\mathrm{NH}_{3}{ }^{+}$surface per unit time. MS2 adsorbed at an initial rate of $60 \mathrm{ng} \cdot \mathrm{cm}^{-2} \cdot \mathrm{min}^{-1}$ (Figure $\mathbf{1 b}$ in the manuscript). This rate corresponds to $5.0 \cdot 10^{8}$ virions $\cdot \mathrm{cm}^{-2} \cdot \mathrm{min}^{-1}$. In an analogous manner, we calculated the number of SRHA assemblies that adsorbed per unit time at the three tested SRHA concentrations. The results of these calculations are compiled in Table S3. The table also shows the approximate number ratios of MS2 to SRHA spheres approaching the SAM- $-\mathrm{NH}_{3}{ }^{+}$surface per unit time. In the first approach, the number ratio varied between 1:1000 and 1:6600 (MS2:SRHA). In the second approach, the number ratio was much lower and varied between 1:55 and 1:334. 
Table S3. Estimated numbers of adsorbing Suwannee River humic acid (SRHA) assemblies per unit time.

\begin{tabular}{|c|c|c|c|c|c|}
\hline \multirow{2}{*}{$\begin{array}{l}\text { SRHA Conc. } \\
\mu \mathrm{g}_{\mathrm{SRHA}} \cdot \mathrm{mL}^{-1}\end{array}$} & \multirow{2}{*}{$\begin{array}{l}\text { Ads. Rate } \\
\mathrm{ng} \cdot \mathrm{cm}^{-2} \cdot \mathrm{min}^{-1}\end{array}$} & \multicolumn{2}{|c|}{ pH $6-I=10 \mathrm{mM}(1 \mathrm{~nm})$} & \multicolumn{2}{|c|}{ pH $6-I=500 \mathrm{mM}(2.7 \mathrm{~nm})$} \\
\hline & & $\begin{array}{l}\text { number of SRHA } \\
\text { assemblies } \cdot \mathrm{cm}^{-2} \\
\cdot \mathrm{min}^{-1}\end{array}$ & $\begin{array}{l}\text { number ratios } \\
\text { MS2:SRHA }\end{array}$ & $\begin{array}{l}\text { number of SRHA } \\
\text { assemblies } \cdot \mathrm{cm}^{-2} \\
\cdot \mathrm{min}^{-1}\end{array}$ & $\begin{array}{l}\text { number ratios } \\
\text { MS2:SRHA }\end{array}$ \\
\hline 0.25 & 2.6 & $5.4 \cdot 10^{11}$ & $\approx 1: 1000$ & $2.7 \cdot 10^{10}$ & $\approx 1: 55$ \\
\hline 0.50 & 4.4 & $9.0 \cdot 10^{11}$ & $\approx 1: 1800$ & $4.6 \cdot 10^{10}$ & $\approx 1: 92$ \\
\hline 2.50 & 15.9 & $3.3 \cdot 10^{12}$ & $\approx 1: 6600$ & $1.7 \cdot 10^{11}$ & $\approx 1: 334$ \\
\hline
\end{tabular}

Estimating fractional surface coverage from final adsorbed masses. Figure S10 shows the calculated adsorbed mass of MS2 viruses as a function of the fractional coverage of the SAM-NH${ }_{3}{ }^{+}$surface, $\theta_{\mathrm{MS} 2}$, using the hydration model of Bingen et al (2008). ${ }^{16}$ The input parameters for the model calculation are shown in Figure S9a (i.e., a diameter of MS2 of $28.8 \mathrm{~nm}$ and a truncated pyramid hydration coat). We used a molecular weight of $3600 \mathrm{kDa}$ and a density of $1.42 \mathrm{~g} \cdot \mathrm{cm}^{-3}$ in the model for MS2. ${ }^{17}$ The calculation were carried out using a Matlab code developed by Bingen et al (2008), ${ }^{16}$ downloaded from http://www.rrichter.net/ (accessed in March, 2015). The model results predict that the jamming limit of the surface is reached at about a final adsorbed mass of $2800 \mathrm{ng} \cdot \mathrm{cm}^{-2}$ and a surface coverage, $\theta_{\mathrm{MS} 2} \approx 0.54$ (Figure S10). This surface coverage is in very good agreement with the theoretical jamming limit for RSA of mono-dispersed spheres of $\theta \approx 0.55 .^{18}$ 


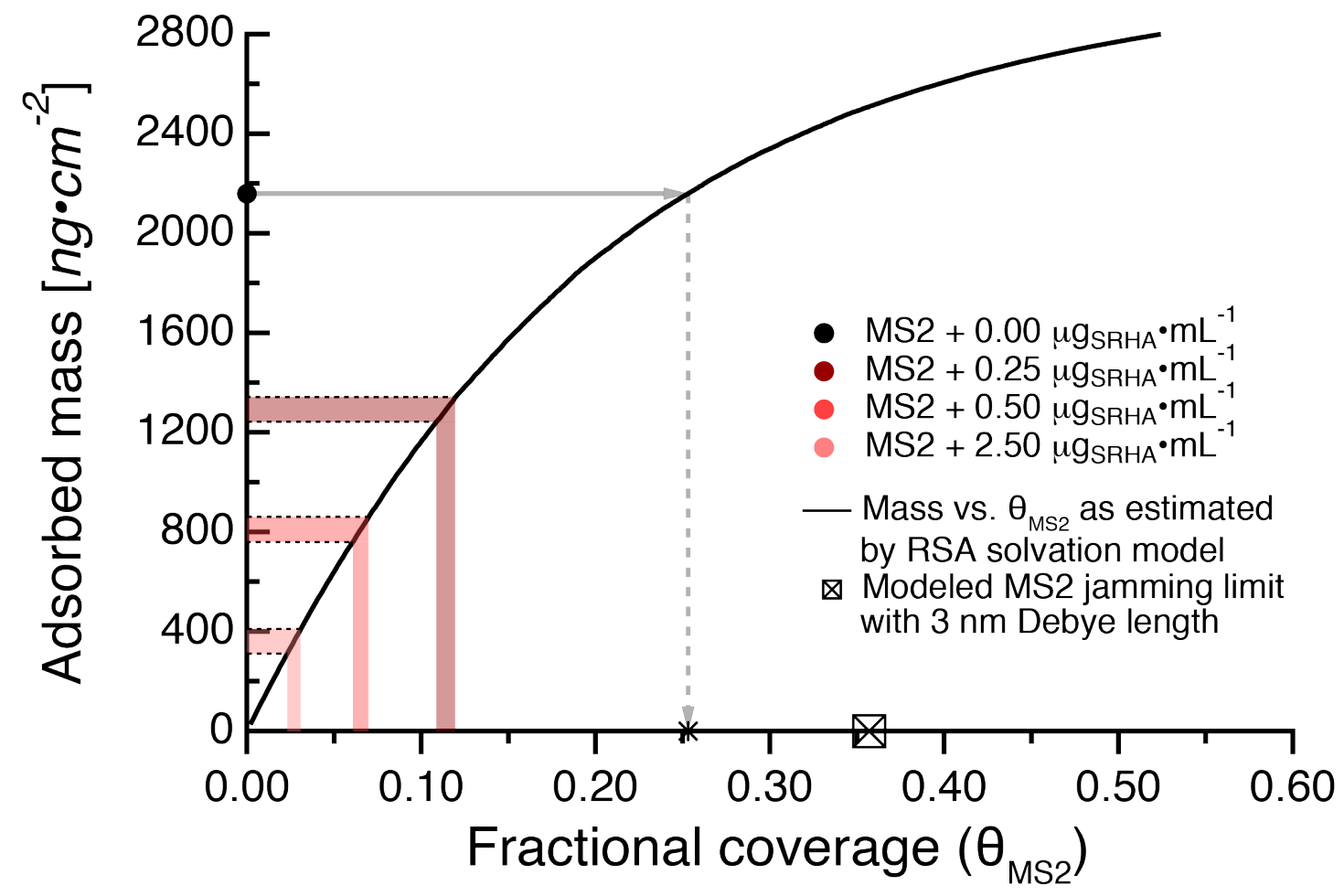

Figure S10. Calculated adsorbed mass of MS2 as a function of the fractional coverage of the adsorbent surface by MS2, $\theta_{\mathrm{MS} 2}$. The calculations were conducted using the hydration model proposed by Bingen et al (2008). ${ }^{16}$

The calculated dependency of adsorbed MS2 mass on the fractional surface coverage of MS2 (Figure S10) can be used to approximate the experimental $\theta_{\text {MS2 }}$ values from the experimentally measured final adsorbed masses. The final adsorbed mass of MS2 in the single-adsorbate experiments was approximately $2200 \mathrm{ng} \cdot \mathrm{cm}^{-2}$. As shown in Figure S10, this mass corresponds to a fractional surface coverage of $\theta_{\mathrm{MS} 2} \approx 0.25$. The experimentally measured final adsorbed masses and $\theta_{\mathrm{MS} 2} \approx$ values were thus significantly smaller than the respective values calculated assuming RSA of MS2 (i.e., $2800 \mathrm{ng} \cdot \mathrm{cm}^{-2}$ and $\theta_{\mathrm{MS} 2} \approx 0.54$; see above).

The smaller experimental than calculated $\theta_{\mathrm{MS} 2}$ values imply that a lower packing density of MS2 on the $\mathrm{SAM}-\mathrm{NH}_{3}{ }^{+}$surfaces was attained experimentally than 
theoretically possible. The smaller experimental $\theta_{\text {MS2 }}$ values can be rationalized based on electrostatic repulsion between MS2 virions. At $I=10 \mathrm{mM}(\mathrm{NaCl})$, the electrostatic field penetrated approximately 3-4 nm away from the negatively charged virus surface into solution (i.e., the calculated Debye length was $3 \mathrm{~nm}$ ). We recalculated the fractional surface coverages of MS2 on the $\mathrm{SAM}-\mathrm{NH}_{3}{ }^{+}$surfaces with a minimum separation distance of $6 \mathrm{~nm}$ between adjacent MS2 virions on the adsorbent surface (i.e., twice the Debye length). Figure S10 shows that the calculations with a minimum separation distance of $6 \mathrm{~nm}$ resulted in $\theta_{\mathrm{MS} 2}$ values of 0.36 and were therefore significantly smaller than the $\theta_{\mathrm{MS} 2} \approx 0.54$ obtained when electrostatic repulsion between MS2 virions was not accounted for.

Figure S11 provides a more detailed analysis of the RSA calculations conducted for MS2 adsorption assuming either no separation distance (panels a, b) or a separation distance of at least $6 \mathrm{~nm}$ (panels $\mathrm{c}, \mathrm{d}$ ) between adjacent MS2 virions on the sorbent surface.

The $\theta_{\mathrm{MS} 2}$ of 0.36 is much closer to the surface coverage estimated from the experimentally measured final adsorbed masses (i.e., $\theta_{\mathrm{MS} 2} \approx 0.25$ ). The remaining discrepancy between modeled and experimental $\theta_{\mathrm{MS} 2}$ values suggests that MS2-MS2 electrostatic repulsion was underestimated by the assumption of a $6 \mathrm{~nm}$ separation distance or that additional factors other than electrostatics lowered the final packing densities of MS2 on the $\mathrm{SAM}-\mathrm{NH}_{3}{ }^{+}$surfaces in the experimental systems. 


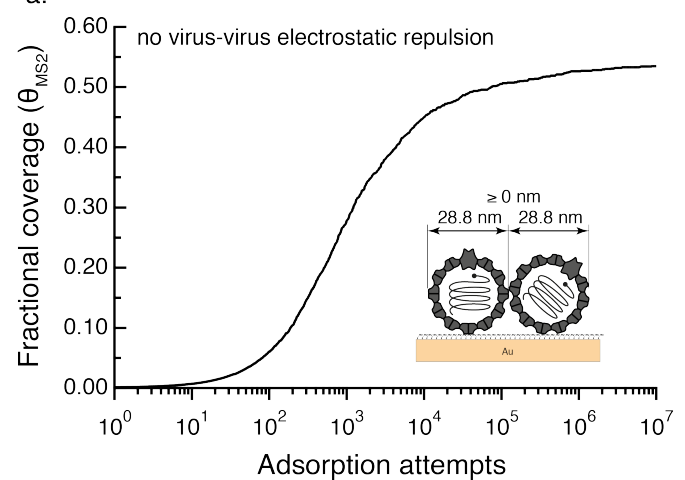

C.

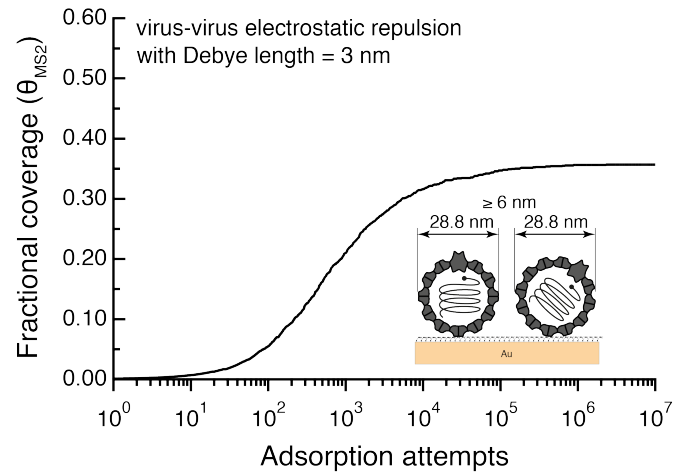

b.

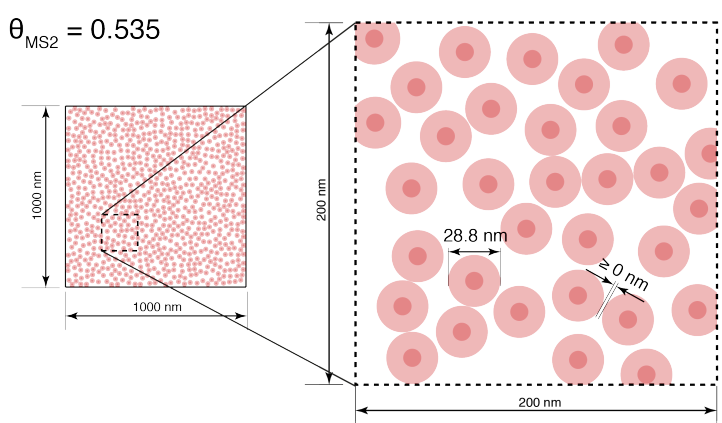

d.

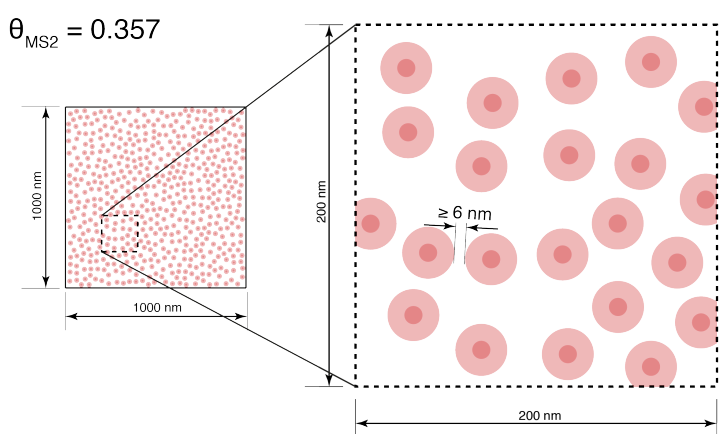

Figure S11. Calculated changes in the fractional coverages of the sorbent surfaces during random sequential adsorption (RSA) of MS2. The virions were modeled as mono-dispersed spheres with diameters of $28.8 \mathrm{~nm}$. Shown are the modeling results assuming that there is no electrostatic repulsion between the MS2 viruses, hence allowing for contact of adjacent MS2 virions in adsorbed states (panels (a) and (b)) and assuming a separation distance of at least $6 \mathrm{~nm}$ between adjacent virions in adsorbed states due to virus-virus electrostatic repulsion (panels (c) and (d)). Panels (a) and (c) show the changes in the fractional surface coverage by MS2 (i.e., $\theta_{\mathrm{MS} 2}$ ) versus the number of simulated adsorption attempts. Panels (b) and (d) depict snapshots of modeled surfaces at the end of the simulations with final coverages of $\theta_{\mathrm{MS} 2}=0.53$ and 0.36 , respectively.

Figure S10 can also be used to estimate the final fractional coverages of MS2 on the SAM- $\mathrm{NH}_{3}{ }^{+}$surfaces in the SRHA-containing coadsorbate systems. We used two approaches to estimate these fractional surface coverages, $\theta_{\mathrm{MS} 2}$. In the first, we directly used the final adsorbed masses obtained in the MS2-SRHA coadsorbate systems (i.e., $\approx 1350,850$, and $400 \mathrm{ng} \cdot \mathrm{cm}^{-2}$ in the presence of SRHA at concentrations of $0.25,0.50$, and $\left.2.50 \mu \mathrm{g} \cdot \mathrm{mL}^{-1}\right)$ to estimate $\theta_{\mathrm{MS} 2}$. This approach neglects mass contributions from SRHA adlayer patches on the SAM- $\mathrm{NH}_{3}{ }^{+}$surfaces to the final 
adsorbed masses. While this assumption may be valid for the coadsorbate system with the lowest SRHA concentration, the SRHA adlayers likely had mass contributions to the final adsorbed mass at the higher two tested SRHA concentrations. The upper dashed horizontal lines in Figure S10 correspond to the first approach to estimate $\theta_{\mathrm{MS} 2}$. In the second approach, we assumed that the SRHA adlayer contributed 100 $\mathrm{ng} \cdot \mathrm{cm}^{-2}$ to the final measured adsorbed masses in the coadsorbate systems. This mass corresponds to that of a complete SRHA adlayer (see Figure 1c in the manuscript) and therefore likely overestimated the actual mass contribution of SRHA to the final adsorbed mass in the coadsorbate system with the lowest SRHA concentration. Conversely, the assumed mass contribution likely was a good approximation for the MS2-SRHA coadsorbate system with the highest SRHA concentration. The lower dashed horizontal lines in Figure $\mathbf{S 1 0}$ correspond to the second approach to estimate $\theta_{\mathrm{MS} 2}$. The two approaches therefore can be considered to provide an upper and a lower estimate of $\theta_{\mathrm{MS} 2}$ in the experimental MS2-SRHA coadsorbate systems. The estimated final MS2 surface coverages are $\theta_{\mathrm{MS} 2} \approx 0.11-0.12,0.06-0.07$, and $0.02-$ 0.03 for the coadsorbate systems with $0.25,0.50$, and $2.50 \mu \mathrm{g}_{\mathrm{SRHA}} \mathrm{mL}^{-1}$. These values are used in Figure 3c,d in the manuscript to compare the simulated with the experimental $\theta_{\mathrm{MS} 2}$ values.

\section{References}

(1) Armanious, A.; Aeppli, M.; Jacak, R.; Refardt, D.; Kohn, T.; Sander, M. Viruses at solid-water interfaces: A systematic assessment of interactions driving adsorption. Environ. Sci. Technol. 2016, 50 (2), 732-743.

(2) Armanious, A.; Aeppli, M.; Sander, M. Dissolved organic matter adsorption to model surfaces: adlayer formation, properties and dynamics at the nanoscale. Environ. Sci. Technol. 2014, 48 (16), 9420-9429. 
(3) Menshikova, A. Y.; Evseeva, T. G.; Skurkis, Y. O.; Tennikova, T. B.; Ivanchev, S. S. Monodisperse carboxylated polystyrene particles: synthesis, electrokinetic and adsorptive properties. Polymer 2005, 46 (4), 1417-1425.

(4) Reviakine, I.; Johannsmann, D.; Richter, R. P. Hearing what you cannot see and visualizing what you hear: interpreting quartz crystal microbalance data from solvated interfaces. Anal. Chem. 2011, 83 (23), 8838-8848.

(5) Golmohammadi, R.; Valegard, K.; Fridborg, K.; Liljas, L. The refined structure of bacteriophage MS2 at $2.8 \AA$ resolution. J. Mol. Biol. 1993, 234 (3), 620-639.

(6) Tomaszewski, J. E.; Schwarzenbach, R. P.; Sander, M. Protein encapsulation by humic substances. Environ. Sci. Technol. 2011, 45 (14), 6003-6010.

(7) Sander, M.; Tomaszewski, J. E.; Madliger, M.; Schwarzenbach, R. P. Adsorption of insecticidal Cry1 Ab protein to humic substances. 1. Experimental approach and mechanistic aspects. Environ. Sci. Technol. 2012, 9923-9931.

(8) Tomaszewski, J. E.; Madliger, M.; Pedersen, J. A.; Schwarzenbach, R. P.; Sander, M. Adsorption of insecticidal Cryl Ab protein to humic substances. 2. Influence of humic and fulvic acid charge and polarity characteristics.

Environ. Sci. Technol. 2012, 9932-9940.

(9) Maroni, P.; Montes Ruiz-Cabello, F. J.; Cardoso, C.; Tiraferri, A. Adsorbed Mass of Polymers on Self-Assembled Monolayers: Effect of Surface Chemistry and Polymer Charge. Langmuir 2015, 31 (22), 6045-6054.

(10) Vermöhlen, K.; Lewandowski, H.; Narres, H. D. Adsorption of polyelectrolytes onto oxides - the influence of ionic strength, molar mass, and Ca 2+ ions. Colloids Surf., A 2000, 163 (1), 45-53.

(11) Norde, W. My voyage of discovery to proteins in flatland . . and beyond. Colloids Surf., B 2008, 61 (1), 1-9.

(12) Haynes, C. A.; Norde, W. Globular proteins at solid/liquid interfaces. Colloids Surf., B 1994, 2 (6), 517-566.

(13) van der Veen, M.; Stuart, M. C.; Norde, W. Spreading of proteins and its effect on adsorption and desorption kinetics. Colloids Surf., B 2007, 54 (2), $136-142$.

(14) Wertz, C. F.; Santore, M. M. Effect of Surface Hydrophobicity on Adsorption and Relaxation Kinetics of Albumin and Fibrinogen: Single-Species and Competitive Behavior. Langmuir 2001, 17 (10), 3006-3016.

(15) Vörös, J. The Density and Refractive Index of Adsorbing Protein Layers. Biophys. J. 2004, 87 (1), 553-561.

(16) Bingen, P.; Wang, G.; Steinmetz, N. F.; Rodahl, M.; Richter, R. P. Solvation effects in the quartz crystal microbalance with dissipation monitoring response to biomolecular adsorption. A phenomenological approach. Anal. Chem. 2008, 80 (23), 8880-8890.

(17) Overby, L. R.; Barlow, G. H.; Doi, R. H.; Jacob, M.; Spiegelman, S. Comparison of two serologically distinct ribonucleic acid bacteriophages I. Properties of the viral particles. J. Bacteriol. 1966, 91 (1), 442.

(18) Adamczyk, Z.; Zembala, M.; Siwek, B.; Warszyński, P. Structure and 
Ordering in Localized Adsorption of Particles. J. Colloid. Interf. Sci. 1990, $140(1), 123-137$. 